Der Vorsitzende: Wir mässen daran festhalten, dą die Bezeichnung ;Himbeersirupersatz" zulässig ist, aber erwünscht ist, wenn wir sagen "mit so und soviel Saccharinlösung hergestellt".

Dr. Bouhon: Es ist doch etwas ganz anderes, ob in einem Himbeersiruperatz der Zucker, oder ob der Himbeermuttersaft ersetzt ist. Das letztgenannte Erzeugnis war schon vor dem Kriege als „künstlicher Himbeersirup “ bekannt, während die neue Bezeichnung „Himbeersirupersatz " besagt, daßß lediglich der Zucker des echten Himbeersirups durch Saccharinlösung ersetzt ist.

Der Vorsitzende: Es melder sich niemand mehr zum Wort, somit wäre dieser Punkt erledigt, wenn Herr Professor Buttenberg nicht noch ein Schlubwort wünscht.

Prof. Buttenberg: Ich fasse also nochmals zusammen: Beim Fruchtsirup, bei dem an Stelle des Zuckers Saccharinlösung zugesetzt worden ist - als Beispiel wähle ich den Himbeersirup - ist die Bezeichnung "Himbeersaft" allein unzulässig. Die Bezeichnung "Himbeersirup-Ersatz" ist zulässig; jedoch ist eine Angabe über die Menge der zugefügten Saccharinlösung erwünscht. Schon mit Rücksicht darauf, daß3 Fruchtsäfte flaschenweisè gehandelt werden, darf das Volumen der zugefügten Saccharinlösung nicht das Volumen des sonst vorhandenen Zackers überschreiten. Fruchtsirup mit 65 Gewichtsteilen zugefügten Zuckers hat etwa das spezifische Gewicht von 1,3-1,4. Im Liter dieses Sirups sind 45 Volumen Muttersaft vorhanden. Demnach ist als höehstzulässige Menge Saccharinlösung ein Zusatz von 55 Teilen anzusehen.

Der Vorsitzende: Wir gehen nun zum folgenden Punkt über: 6 . Was ist Marmelade und Kunstmarmelade im Sinne der Höchstpreisverordnungen, wie sind ohne Obst und Obstrückstände hergestellte marmeladenähnliche Zubereitungen (Brotaufstriche) zu beurteilen? Ich bitte Herrn Professor Butten berg, uns darüber zu berichten.

\title{
Was ist Marmelade und Kunstmarmelade im Sinne der Höchstpreisverordnungen? Wie sind ohne Obst oder Obst- lückstände hergestellte marmeladenähnliche Zubereitungen (Brotaufstriche) zu beurteilen?
}

\author{
Berichterstatter: Prof. Dr. P. Buttenberg-Hamburg.
}

Die Heidelberger Beschlüsse von 1909 haben es ermöglicht, die seiner Zeit im Verkehr mit Marmelade bestehenden Übelstände wirksam zu bekämpfen. Durch den jetzt im Kriege notwendig gewordenen Erlaß der Höchstpreisverordnung nebst Sorten. bezeichnung werden die Heidelberger Beschlüsse nicht aufgehoben, wohl aber hat der Begriff Marmelade, den Kriegsverhältnissen entsprechend, eine gewisse Erweiterung erfahren. Die Sortenbezeichnung bietet manche Vorteile und es dürfte daher auch zu erwägen sein, ob diese Bestimmung nach dem Kriege beizubehalten ist. Ein wirksames Mittel, die großen Mißstände zu beseitigen, die sich beim Verkehr mit marmeladenähnlichen Zubereitungen einschließlich Marmeladenhalbfabrikaten eingestellt haben, ist die Höchstpreisverordnung nicht.

Zu Marmelade einschließlich Kunstmarmelade, welche unter die Höchstpreisverordnung fallen, können alle möglichen Obstsorten, Fruchtrückstände; Rhabarber, Mehl, Kürbis, Tomaten, Kartoffeln, Rüben usw. verarbeitet werden, sodaß e in erheblicher Gehalt an zugefügtem Zucker ein Hauptmerkmal und zugleich Unterscheidungsmittel von vielen erwünschten und unerwünschten marmeladenartigen Zubereitungen, mit denen uns die Kriegsindustrie in so reichlichem Mabe bedacht hat, bildet. Diese marmeladenähnlichen Zubereitungen, von denen viele nur iṇ bezug auf Aussehen eine gewisse Ähnlichkeit mit Marmelade aufweisen, lassen sich in folgende Hauptgruppen einteilen: 
1. Fruchtmark und Fruchtmus,

2. Rübenmark,

3. Zubereitungen aus Carragheenmoos,

4. Zubereitungen aus Gelatine und Agar.

Die Friedensmarmelade war sehr reich an zugesetztem Zucker. Die großen Ansprüche, welche jetzt an unsere Zuckervorräte gestellt werden, machen einen sparsamen Verbrauch notwendig. Die Reichskanzler-Bekanntmachung vom 24. Juni 1916 verbietet bei der gewerbsmäBigen Herstellung von gewissen Obstzubereitungen $\mathrm{z}$. $\mathrm{B}$. eingemachten Früchten die Verwendung von Zucker, gestattet dagegen bei Marmeladen einen Gehalt an zugefügtem Zucker von nicht mehr als 50 vom Hundert des fertigen Produktes. So kommt es, daß die Kriegsware vielfach weniger steif ist, zumal wenn der Fabrikant dies nicht durch Zusatz von Obst mit größerer Gelierfähigkeit ausgeglichen hat. Neuerdings ist mit Rücksicht auf Haltbarkeit und Verbilligung der Ware bei einer Reihe von Marmeladen auch ein Zuckerzusatz von $60 \%$ vorgesehen.

Die Höchstpreisverordnung vom 14. Dezember 1915 sieht für Sorte I Einfruchtmarmelade ausschließlich Apfelmarmelade Höchstpreise nicht vor, da für die Massenernährung die Sorte II bis $\mathrm{V}$ in Betracht kommen. Zur Herstellung der Sorte I ist den Fabrikanten Zucker nur in beschränkter Menge zur Verfügung gestellt worden. Erst die spätere Bekanntmachung, erlassen von der Kriegsgesellschaft für Obstkonserven und Marmeladen am 14. August 1916, setzt für die hauptsächlichsten Marmeladen der Sorte I und zwar für Erdbeer-, Himbeer-, Johannisbeer-, Kirsch-, Heidelbeer-, Stachelbeer-, Pflaumen- und Zwetschen-Marmelade Preise fest. Eine Reihe von Früchten finden dort keine Beachtung, von denen nur Orangen, Pfirsiche und Aprikosen genannt werden sollen.

Die Art, wie Äpfel bei Marmeladen verwendet werden können, ergibt sich klar aus dem Wortlaut des Sortenverzeichnisses.

Birnen werden meist mit anderen Früchten verarbeitet. Reine Birnenmarmelade, die $a b$ und zu vorkommt, dürfte der Apfelmarmelade (Sorte III) gleichzustellen sein.

Fruchtrückstände, die bei den Sorten III, IV und V zulässig sind, dürfen naturgemäß, wie dies in den Heidelberger Beschlüssen zum Ausdruck kommt, nicht aus wertlosem, mit Wasser ausgelaugten Material bestehen.

Rhabarber, der in der Konservenindustrie etwa dem unansehnlich gewordenen Obste gleichgestellt wird, darf nach der schon zuvor genannten Bekanntmachung vom 14. Angust 1916 lediglich bei der Herstellung der Sorten III, IV und V Verwendung finden.

Bei der Sorte IV, Kunstmarmelade, läßt sich manches Kriegsprodukt unterbringen, das an sich noch minderwertiger ist als Sorte $V$. Ich nenne hier die gezuckerten Produkte aus verkleisterter Stärke mit etwas Aroma und Farbe versetzt. Auch Kürbismarmelade dürfte zu Sorte IV zu rechnen sein.

Eine Verarbeitung von Wurzelgewächsen (Kartoffeln und Rüben) (Sorte V) haben wir vor dem Kriege bei den für den Verkehr bestimmten Marmeladen nicht gekannt. Schon auf der vorjährigen Hauptversammlung haben Besprechungen darüber stattgefunden, wie der Wortlaut der Sorte $V$ aufzufassen ist. Es ist erörtert worden, ob eine Marmelade als Sorte $V$ anzusehen ist, wenn Rüben oder Kartoffeln zugesetzt sind, oder ob das zweite Wurzelgewächs gleichzeitig vorhanden sein muß. Wir sind uns wohl jetzt darüber einig, daß Marmelade mit Zusatz von Rüben unter Sorte V fällt, auch wenn eine Beigabe von Kartoffeln nicht erfolgt ist. Selbst bei

N. 18 . 
Sorte V ist ein gewisser Gehalt an Fruchtmasse oder Fruchtrückständen erforderlich, denn die Verordnung sagt nicht „Marmeladen aus Rüben und Kartoffeln", sondern wählt den Wortlaut „Marmeladen unter Zusatz von Rüben und Kartoffeln“.

Vogelbeeren sind schon früher in Haushaltungen zu Marmelade verarbeitet worden; jetzt dient der Vogelbeersaft dazu, an sich fade schmeckende Produkte etwas schmackhafter zu machen.

Stärkesiruphaltige Marmeladen sind im Kriege bald verschwunden, weil der Mangel an Kartoffeln die Herstellung von Stärkesirup unterbunden hat.

Stärke ist bäufig in Marmeladen angetroffen worden. Beim Auffinden von Stärke muß man sich über deren Art und Menge näher unterrichten, da ganz verschiedene Sorten vorliegen können. Der stark verkleisterte Zustand der Stärke trägt nicht dazu bei, die Untersuchung über die Herkunft zu erleichtern. Kleine Mengen Stärke können von verarbeiteten unreifen Äpfeln und Birnen herrühren. Reich an Stärke ist Bananenmarmelade. Das Ausgangsmaterial, die getrockneten Bananen, ist zur Zeit längst verbraucht. Meist bezweckt der Stärkezusatz, wasserreichen, fruchtmarkarmen Fabrikaten eine steife Beschaffenheit zu verleihen. Derartige Erzeugnisse, auch wenn der Zusatz in Form von Kartoffelmehl erfolgt ist, sind als Kunstmarmelade Sorte IV anzusprechen. Ist die Kartoffelstärke nicht in Form von Kartoffelmehl, sondern in Form von zerkleinerten Kartoffeln vorhanden, so ist das Fabrikat unter Sorte $\mathrm{V}$ einzureihen.

Der Auffassung, daß der Zusatz von mehreren Prozent Stärkemehl als erlaubtes Geliermittel bei Sorte III oder sogar II anzusehen ist, können wir uns nicht anschließen, auch wenn Leitsatz 10 der Heidelberger Beschlüsse in wenig glücklicher Form von "Agar, Gelatine und ähnlichen Geliermitteln" spricht. Damals hat man an Tragant gedacht.

Von den Marmeladen müssen wir sehr wohl das Fruehtmark, das Halbfabrikat der Marmelade, und das weiter eingedickte Fruchtmus unterscheiden. Beide fallen nicht unter die Höchstpreisverordnung für Marmeladen. Infolge des Zuckermangels kommt viel Fruchtmark von ganz verschiedener Güte in den-Kleinhandel. Fruchtmark und Fruchtmus können sich teurer stellen als Marmelade. Es hängt dies $a b$ vom Preise des Zuckers und der Früchte. Bei wertvollem Fruchtmark kann Zucker die Wirkung eines Verbilligungsmittels ausüben. Bei stark wasserhaltigem Fruchtmark, Fruchtrückständen, Rüben und Kartoffeln ist das Umgekehrte der Fall.

Wie sich die Preise von Fruchtmark, Fruchtmus und Fruchtmarmelade zueineinander stellen, kann am besten bei den Pflaumenzubereitungen vor Augen geführt werden. 125 Pfund frische Pflaumen geben 100 Pfund Pflaumenmark, daraus gewinnt man durch mehrstündiges Eindampfen etwa 25 Pfund Pflaumenmus. Bei einem Kostenpreis von $15 \mathrm{Mk}$. für 100 Pfund Pflaumen würde sich demnach das Material an Pflaumen für $0,5 \mathrm{~kg}$ Pflaumenmark auf $0,19 \mathrm{Mk}$. und für $0,5 \mathrm{~kg}$ Pflaumenmus auf $0,75 \mathrm{Mk}$. stellen. Wenn man 125 Pfund frische Pflaumen mit 50 Teilen Zucker verarbeitet, erhält man 100 Pfund Pflaumenmarmelade. Bei einem Zuckerpreise von $34 \mathrm{Mk}$. für 100 Pfund würden „die Zutatenkosten $0,36 \mathrm{Mk}$. für $0,5 \mathrm{~kg}$ Pflaumenmarmelade betragen. In der nachfolgenden Zusammenstellung sind auch die Friedenspreise eingefügt. Als Kosten sind überall nur die Ausgaben für Pflaumen bezw. für Pflaumen und Zucker in Ansatz gebracht: 


$$
\text { Preise der Zutaten } \begin{cases}\text { a) Kriegspreise: } & \text { b) Friedenspreise } \\ \text { Pflanmen 100 Pfund }=15 \mathrm{M} . & =4 \mathrm{M} . \\ \text { Zucker 100, } \quad=34 \mathrm{M} . & =28 \mathrm{M} .\end{cases}
$$

\begin{tabular}{|c|c|c|c|c|}
\hline Fabrikat & Ausgangsmaterial & Ausbeute & $\begin{array}{l}\text { Kriegspreis } \\
\text { fürr } 0,5 \mathrm{~kg}\end{array}$ & $\begin{array}{l}\text { Friedenspreis } \\
\text { für } 0,5 \mathrm{~kg}\end{array}$ \\
\hline & 125 Pfund Pflaumen. & 100 Pfund & $0,19 \mathrm{M}$ & $0,05 \mathrm{M}$. \\
\hline Mus & 125 Pfund Pflaumen. . & 25 & 0,75 & 0,20 \\
\hline Marmelade & 125 Pfund Pflaumen +50 Pfund Zucker & 100 & 0,36 & 0,19 \\
\hline
\end{tabular}

Die vorstehenden Berechnungen sind an der Hand von Angaben ausgefübrt, die ich der Liebenswürdigkeit eịes norddeutschen Fabrikanten verdanke.

Jedenfalls stellt sich jetzt im Kriege das Pflaumenmus ganz wesentlich teurer als Pflaumenmarmelade, für welche unter Sorte $I$ ein Preis von 0,64 Mk. festgesetzt ist. Pflaumenmus und Pflaumenmarmelade können daher im Preise nicht gleich sein. Dies bezieh sich naturgemäß nur auf ein unverfälschtes Pflaumenmus mit einer Trockenmasse von etwa $50-60 \%$. Was wir im Kriege unter der Bezeichnung Pflaumenmus angetroffen haben, war meist ein dünnes, sehr wasserreiches Pflaumenmark, das bei einer Trockenmasse von etwa 12-15\% vielfach mit weniger wertvollem Obstmark (Apfel, Birnen) oder sogar noch mit Rüben gestreckt war. Pflaumenmus wird viel in Haushaltungen hergestellt und kommt von dort aus wohl auch in den Verkehr. Das gewerbsmäßige Herstellen von Pflaumenmus aus frischen und getrockneten Früchten ist durch die Bekanntmachung vom 27. November 1916 verboten. Der Zweck dieser Bestimmung ist, die Pflaumenbestände in möglichst gestreckter Form als Marmelade und musartige Zubereitung zu verbrauchen.

Apfelmarmelade (Sorte III) wird mit $0,50 \mathrm{Mk}$. für $0,5 \mathrm{~kg}$ bezahlt; für Apfelmus ist dagegen am 17. April 1917 ein Preis von 1,30 Mk. für die 1/1-Dose $(=1 \mathrm{~kg})$ festgesetzt worden. Die Abgabe des letzteren ist jedoch nur an Lazarette, Sanatorien und Krankenanstalten gestatitet.

Daß wir, um das erforderliche Aufstrichmaterial zu beschaffen, zu den $R$ üben greifen müssen, wird wohl allgemein als bittere Kriegsnotwendigkeit empfunden. Mit Mohrrüben bat man scbon früher im Haushalte Marmelade und eingemachte Früchte gestreckt. Auch marmeladenartige Zubereitungen aus sogenannten roten Rüben waren bekannt. Runkelrüben hat man ebenfalls gelegentlich verwendet. Das Hauptmaterial zur Herstellung des Rübenmarkes bildete bisher die Kohlrübe, die uns im letzten Winter über den Kartoffelmangel hinweggeholfen hat.

Abgesehen von der Verarbeitung zur Marmelade ist das Rübenmark als solches mit Saccharin versetzt in Massen vertrieben worden. Um es ansehnlicher und schmackbafter zu machen, hat es Zusätze von Farbe (gelb, rot), Fruchtsäuren, A romastoffen (Zitronen-, Orangenschalen, Fruchtäther), auch Beimengungen von Fruchtmark (Apfel, Pflaumen, Preißelbeeren u. dergl.) erhalten. Zweifellos ruft sehon der eigenartige Geruch der Rüben bei vielen Personen Widerwillen hervor. Ein bitterer Geschmack hat häufig Veranlassung zu Klagen gegeben. Der bittere Geschmack wird durch den Kopf und die Schale der Kohlrübe hervorgerufen. Um Kohlrüben zu entbittern, wird Dämpfen, Behandeln mit kohlensaurem Alkali, auch Einlegen in Essig vorgeschlagen, Derartige Verfahren dürfen nicht mit einem beträchtlichen Verluste an wasserlöslichen Stoffen (Zucker) verknüpft sein. Auch das ungenügende Entfernen 
von schadhaften oder direkt angefaulten Stellen beeinträchtigt Geschmack und Haltbarkeit. Hoher Sàndgehalt läßt auf ungenügendes Waschen der Rüben schließen.

Das Rübenmark des Handels enthält etwa 83-93\% Wasser. Die Bezeichnung von Rübenmark war vielfach irrreführend und der Preis unangemessen hach. Wenn man in einem derartigen Rübenmus 50 Teile Wasser durch 50 Teile Zucker ersetzt, so erhält man eine Marmelade Sorte $\mathrm{V}$, die mịt $0,35 \mathrm{Mk}$. für $0,5 \mathrm{~kg}$ vertrieben werden darf. Die Rübenmussorten dürfen diesen Preis nicht überschreiten.

Die Knappheit an vollwertigen Marmeladen war die Veranlassung, im Dezember 1916 das sogenannte Krieg s m us einzuführen und durch die Gemeindeverwaltungen zum Preise von $0,55-0,60 \mathrm{Mk}$. zu vertreiben. Dieses Präparat besteht aus etwa $20 \%$ Obst, $30 \%$ Rüben und 50\% Zucker. Über den Geschmack ist viel geklagt worden. Vielfach haben sich diese Klagen aber auch auf gewöhnliches ungezuckertes Rübenmark bezogen, das unter der irreführenden Bezeichnung Kriegsmus vertrieben wurde. Nach der Bekanntmachung vom 5. August 1916 finden auf Marmeladen, die mit Genehmigung der Kriegsgesellschaft für Obstkonserven und Marmeladen abgesetzt werden, die in der Bekanntmachung vom 14. Dezember 1915 festgesetzten Höchstpreise keine Anwendung. Es kann daher davon abgesehen werden, Betrachtungen darüber anzustellen, ob das Kriegsmus unter Sorte $V$ fällt. $E s$ ist beabsichtigt, in Zukunft die Kohlrüben im Kriegsmus durch Runkelrüben, Kürbis und Mohrrüben zu ersetzen. Wir wollen hoffen, daß in diesem Jahre soviel Obst und Obstfabrikate zur Verfügung stehen und in brauchbarem Zustande erhalten bleiben; daß nicht nur der Bedarf des Heeres, sondern auch der Zivilbevölkerung möglichst ausreichend durch steckrübenfreie Fruchtmarmelade gedeckt werden kann.

Im großen Umfange ist Carragheenmoos, das Ausgangsmaterial für Salatölersatz, zur Herstellung von marmeladenähnlichen Zubereitungen verwendet worden. Einzelne dieser Produkte bestanden lediglich aus mit Carragheenmoos und etwas Stärkemehl verdicktem Wasser mit Zusatz von Farbe, Saccharin und Aromastoffen (Fruchtäther, Orangenschalen $u$. dergl.); vielfach war auch etwas Steckrübenmark zugefügt worden. . Der Wassergehalt betrug etwa $86,0-93,0 \%$. Der Vertrieb erfolgte unter der Bezeichnung "Brotaufstrich", „Kunsthonig“, „Honigmus“, „Orangenmarmelade“, "Ananasmarmelade", „Marmelade“ u. dergl. zu hohen Preisen.

Steckrüben mark, mit Agar und Gelatine verdiekt, ist ebenfalls vorgekommen. Die besprochenen Fabrikate aus Carragheenmoos, Gelatine und Agar können selbst als Kunstmarmelade (Sorte IV) nicht mehr angesehen werden. Die Beurteilung auf Grund des Nahrungsmittelgesetzes und auf Grund der Bekanntmachung gegen irreführende Bezeichnung bietet keine Schwierigkeiten.

Mit dem Worte, "Brotaufstrich" als Bezeichnung einer Handelsware wird viel Unfug getrieben. Dieser Name besagt, daß eine Zubereitung vorliegt, die nicht nur infolge ihrer Konsistenz geeignet ist, das in Scheiben geschnittene Brot zu bestreichen, sondern vor allem auch dazu dient, die Schmackhaftigkeit und den Genußwert des Brotes zu erhöhen. Jetzt im Kriege wird die Benennung Brotaufstrich als selbständige Handelsbezeicknung bei vielen Präparaten' benutzt, die wohl eine Streichbarkeit aufweisen, aber vielfach ohne jeden Nähr- und Genußwert sind. Wenn mit Gelatine, Carragheenmoos u. dergl. verdicktes Wasser als „Brotaufstrich“ vertrieben wird, so ist hierin eine Irreführung zu erblicken. Man soll daher das Wort „Brotaufstrich" als Bezeichnung einer bisher nicht bekannten Handelsware grundsätzlich zurückweisen. Bei der Auswahl des Namens „Brotaufstrich" hielt man sich be- 
rechtigt, unabhängig von den Bestimmungen über Marmelade, hohe Preise fordern zu können. Unerwünschte Bezeichnungen, die sich im Kriege einbürgern, werden auch später wieder auftauchen und dann voraussichtlich schwieriger auszurotten sein.

Bei der Anferligung von marmeladenartigen Zubereitungen spielt auch Gelatine eine Rolle. Als Verdickungsmittel für Fruchtmark ist Gelatine wenig geeignet. Dagegen ist als "Himbeergelee", "Brotaufstrich", "Marmelade" u. dergl. in großen Massen ein Produkt zu teuren Preisen verkauft, das aus $58 \%$ Wasser, $2 \%$ Gelatine, etwas Anilinfarbe und Fruchtäther bestand. Daß mit einem Zusatz von etwa $2 \%$ eine fast schnittfeste Gallerte erzielt worden ist, läßt darauf schließen, daß tatsächlich Gelatine und nicht gewöhnliches Leimpulver, das man so häufig bei dem sogenannten Fruchtgeleepulver antrifft, Verwendung gefunden hat. Die Übelstände beim Verkehr mit Gelatine sind bekannt, sie baben schon Anfang des Jahres zur amtlichen Warnung Veranlassung gegeben. Nebenbei sei bemerkt, daß diese Leimpulver außer am Geruch, Geschmack und Farbe an der geringen Gelierfähigkeit - zu bestimmen in 2, 4, 6 und 10\%-iger Lösung - und dem niedrigem Schmelzpunkt - zu bestimmen an 10\%-iger Lösung, schräg erstarrt im Reagensglas —, ferner an dem hohen Gehalte an Azche und meist auch an schwefliger Säure zu erkennen sind. Auch gute Speisegelatine ist fast durchweg geschwefelt. Während einwandfreies Gelatinepulver nach unseren früheren Untersuchungen ${ }^{1}$ ) etwa $0,02-0,14 \%$ schweflige Säure aufweist, werden in den gepulverten Leimsorten Mengen bis 0,5 und 1,0\% gefunden.

Viel Marmelade wird in Haushaltungen dadurch verdorben, daß das Unterbringen der Früchte und das Einkochen im verzinkten Kessel erfolgt. Dieselbe Erscheinung macht sich auch bei Fruchtsäften unliebsam bemerkbar. Recht erhebliche Mengen von wertvollem Material sind anch noch im vierten Kriegsherbst dabei verloren gegangen. Die Warnungen in den Zeitungen werden leider nicht genügend beachtet. Auch in der Anweisung über das Einkochen von Obstzubereitungen müssen Belehrungen über die Verwendung von verzinkten Geräten aufgenommen werden.

Die Unterbringung der Marmeladen in Behältern aus Schwarzblech, besonders wenn deren Vernierung beim Eintritt des Gärens schadhaft wird, hat Verfärbung und Aufnahme von Eisen, das sich durch adstringierenden Geschmack bemerkbar machen kann, zur Folge. Die Unterbringung von Marmelade im Pappkarton hat sich bewährt.

Wenn Obst direkt mit einer genügenden Menge Zucker zur Marmelade eingekocht wird, ist der Zusatz eines Konservierungsmittels nicht unbedingt erforderlich. Da in den Fabriken das Obst in Form von Fruchtmark aufgespeichert wird, ist die Haltbarmachung durch Chemikalien vielfach üblich. $\mathrm{Zu}$ diesem $Z$ weeke wird u. a. auch schweflige Sä ure in Form wässeriger Lösung in Mengen von etwa 0,1 bis $0,3 \%$ verwendet. Die zugefügte schweflige Säure oxydiert sich zum großen Teile beim Lagern und muß vollständig vor der Zuckerbeigabe durch Erhitzen des Fruchtmarkes beseitigt werden. Fertige Marmelade soll frei von schwefliger Säure sein. Marmelade mit nachweisbaren Mengen von schwefliger Säure haben wir im Kriege nicht angetroffen.

Die an sich schätzenswerten Bestrebungen, Fremdwörter auszumerzen, haben dahin geführt, an Stelle von "Marmelade" das Wort „Schachtelmus" zur Anwendung zu bringen. Es wird damit auf die portugiesische Herkunft (märmelo = Quitte, marmelada

\footnotetext{
1) Diese Zeitschrift 1906, 12, 408-409.
} 
= eingedicktes, in Schachteln gegossenes Quittenmus) hingedeutet. Diese Verdeutschung wird dem großen Publikum nicht erklärlich sein, da man bei uns ein derartiges Produkt nicht kennt. Die Berechtigung läßt sich auch nicht dadurch begründen, daß einzelne Fabrikanten Marmelade in den Butterdosen nachgebildeten Schachteln vertreiben. Der ab und $\mathrm{zu}$ in Tageszeitungen gemachte Vorschlag, das Wort Marmelade durch Mus zu ersetzen, würde nur eine Verwirrung in der Benennung unserer zubereiteten Nahrungsmittel herbeiführen.

Bei der Überwachung des Verkehrs mit Marmelade und marmeladenähnlichen Zubereitungen hat sich als wirksames Mittel, die Verbraucher vor dem Inverkehrbringen minderwertiger Ware zu schützen, die möglichst zeitig einsetzende Fabrikkontrolle erwiesen.

\section{Disk us sion.}

Der Vorsitzende: Ich stelle die Ausführungen zur Diskussion.

Prof. W. Fresenius: Ich möchte nur die Frage des Zinkgehaltes berühren. Ich habe mich bemüht, die Hausfrauen öffentlich darauf hinzuweisen, dak sie das Einkochen in verzinkten Kesseln unterlassen sollen. Als die Kupferwaschkessel eingezogen wurden, benutzte man als Ersatz meist verzinkte Kessel. Den Hausfraten kam dabei nicht zum Bewuftsein, dáb sich diese Waschkessel nicht zum Marmeladeneinkochen eignen. Deshalb sind sie vielfach hierzu benutzt worden, wodurch relativ große Mengen solcher Obsterzeugnisse hergestellt wurden, die ungenießbar waren und verschiedentlich erhebliche Gesundheitsstörungen bedingten. Einmalige Warnungen finden natürlich nicht die notwendige Beachtung. Deshalb sind die Bestrebungen durchaus am Platze, auf das Unzulässige der Benutzung verzinkter Kessel immer wieder hinzuweisen. In erster Linie ist es notwendig, das Publikum aufauklären. Es ist dringend erwünscht, daßs zu den Zeiten, in denen Marmelade eingekocht wird, von den Behörden die Hausfrauen darauf hinzuweisen sind, dafs sie die Benutzang von verzinkten Kesseln zur Herstellung von Obsterzeugnissen unterlassen. Ich weif $j a$, wie schwer es ist, Unkenntnis zu bekämpfen, aber es ist deshalb doppelt notwendig, diese Hinweise stots erneut zu bringen.

Prof. Härtel: Wir müssen daran festhalten, daß̧ Marmeladen Zubereitungen aus frischen Frächten und Zucker sind. Die Herstellung von Rübenmarmelade and die Bezeichnung "Rüben"-Marmelade ist, da Rüben keine Früchte sind, unzulässig. Vom Schöffengericht Leipzig ist eine Verurteilung erfolgt, weil der Beschuldigte sein hauptsächlich aus Rüben bestehendes Erzeugnis als "Rüben “-Marmelade in Verkehr gebracht hatte; die Bezeichnung "Marmelade* sei aber für dies Produkt nicht zulässig, da Marmeladen aus Frucht und Zucker zu bestehen hätten und nur "Zusätze" von Rüben usw. unter Kennzeichnung statthaft seien. Da der Angeklagte sich bei dem Urteile beruhigt hat, so liegt keine Entscheidung einer höheren Instanz vor. Dies ist $z u$ bedauern, jedenfalls müssen wir aber in Zukunft streng daran festhalten, daß die normalen Bestandteile für Marmeladen nur Frucht und Zucker sind. -- Was nun die Angabe, "Agar, Gelatine und ähnliche Geliermittel ${ }^{\star}$ in den Heidelberger Beschlüssen betrifft, so hatten wir unter ,ähnliche Geliermittel" Leim, Tragant u. dergl. im Auge. Wir wollten dies nicht besonders sagen, um hierdurch die Anregung zur Verwendung zu geben. Jedenfalls haben wir unter "ähnliche Geliermittel" nicht an Stärke gedacht. - Bezüglich der Konservierung mit schwefliger Säure möchte ich erwähnen, daß auch mir bekannt ist, ,daß von den meisten Konservenfabriken diese Konservierung der Früchite jetzt benutzt wird. Wenn das mit schwefliger Säure behandelte Fruchtmark vor Herstellung der Marmeladen gekocht wird, so verflüchtigt sich die noch als solche vorhandene schweflige Säure und in den Marmeladen ist meist keine oder nur Spuren schwefliger Säure nachweisbar. Ich möchte aber darauf hinweisen, dab durch diese Konservierung die Untersuchungsbefunde beeinflufat werden; die Alkalität der Asche und die Alkalitätszahlen gehen herunter. Ich habe früher gezeigt, daf man die Verwendung von geschwefeltem Dörrobst mit Hilfe der niedrigen Alkalitätszahlen nachweisen kann, z. B. gelang diès bei Aprikosenmarmelade śelur gut. Da zur Zeit keine geșchwefelten Aprikosen im Handel zu haben sind, kommt dies jetzt nicht in Frage. Wenn die Herren jetzt niedrige Werte für die Alkalitätszahl finden; so ist dies anf die Konservierung der Früchte mit schwefliger Säure zurückzuführen. "Von einer Seite wird nun empfohlen, die schweflige Säure vor Verwendung des Fruchtmarkes mit Wasserstoffsuperoxyd zu oxydieren und dann mit Natriumearbonat zu neutralisieren. Dies kann ich nicht empfehlen. Ich kann Ihnen auch die Mengen schwefliger Säure, welche zur Konservierung der Frî́chte verwendet werden, angeben, z. B. sollen für $100 \mathrm{~kg}$ Kirschmárk etwa $2,2516-8 \%$-iger 
schwefliger Säure genommen werden. Würde diese Menge oxydiert und neutralisiert, so würde in $1 \mathrm{~kg}$ Marmelade dann eine Menge Sulfat onthalten sein, welche bis $\mathrm{zu} 7,9 \mathrm{~g}$ Natriumsulfat entspricht. Dies ist natürlich ein zu großer Sulfatgehalt und ich halte daher die Behandlung mit Wasserstoffsuperoxyd und die nachherige Neutralisation nicht für zulässig. Von den mir bekannten Fabriken wird auch kein Wasserstoffsuperoxyd verwendet, sondern das Mark wird nur kräftig gekocht, wodureh die noch vorhandene schweflige Säure entweicht. Ob durch die Behandhung mit schwefliger Säure noch andere Veränderungen der Fruchtbestandteile in Betracht kommen, kann ich noch nicht sagen. Ich bin mit diesbezüglichen Versuchen beschäftigt, die Versuche sind aber noch nicht abgeschlossen.

Geheimrat Herzfeld: Die Reichsstelle für Obstverteilung hat erklärt, daß sie den Marmeladenfabriken 5 Millionen Zentner Obst überwiesen habe und glaubt, das damit die Bevölkerung genügend versorgt sei. Wenn wir aber bedenken, daf uns gegenüber den vergangenen Jahren 1 Million Tonnen Fett, das aus dem Ausland hereinkam, fohlt, dann ist es ganz klar, daß 5 Millionen Zentner Obst kaum etwas bedeuten können für die Versorgung der Bevölkerung mit Streichmitteln. Es kommt diese Menge 48 Millionen Tonnen Zucker gleich. Wenn wir annehmen, daß die Produkte $70 \%$-ig sind, so kommen wir auf einen Bedarf von 50-60 Millionen Zentner als Ersatz für das ausländische Fett, aber von den dünnen Aufstrichprodukten brauchten wir wohl 100 Millionen Zentner. Hierzu kommt noch, daf wir eine Mikernte an Obst haben. Schon seit Wochen und Monaten sind Kommunalverbände an mich herangetreten mit der Frage, wie sie Ersatzstoffe aus Rüben herstellen können. An Kunsthonig können wir eine gewisse Menge herstellen. Wonn wir die vorige Ernte an Zuckerrüben zugrundelegen - wir verfüttern aber heute mehr Räben als im Vorjahr - dann können wir auf 27 Millionen Zentnor Zucker rechnen und können 10 Millionen davon für die Herstellung von Kunsthonig verrwenden. Wir müssen also bei dem Mangel an Obst notwendig zu den kuben als Ersatzmittel greifen. Ieh habe auf Veranlassung der Reichsznckerstelle nach einem Vorschlage von Professor Müller.Halle Rüben bei 5 Atmosphären Druck zu dämpfen versucht und konnte ein sehr feines Mus herstellen. Die Räben werden gekocht, dann durch feinste Passiermaschinen getrieben, mit Salzsäare angesäuert und mit $10 \%$ Apfelmark versetzt.

Dr. Cantzler: Der Zinkgehalt kommt, wie schon erwähnt, aus Unkenntuis der Hausfrauen in das Mus. Es ist nun eine andere Frage akut geworden, ob wir nämlich nich helfen können, das Zink aus dem Mus wieder zu entfernen. Bei mir war eine Hausfrau, die einen ganzen Zentner Mus hatte, das Zinkgehalt aufwies. Ich konnte nun das Zink elektrolytisch bis auf $0,1 \%$ wieder entfernen. Es sind jetzt Versuche im Gang, damit wir einen Weg finden, das Mus wieder genufsähig zu machen. Es wäre empfehlenswert, auch andererseits Versuche anzustellen, $o b$ man das Mus regenerieren und zinkfrei machen kann. Ich habe 2 Kohlenpole in das Mus hineingesteckt und dann einen elektrischen Strom durchgeschickt. Der eine Pol hatte sich in 10 Minuten mit Zink bedeckt. Ich habe keine Bedenken, dieses Mus als genubfähig zu bezeichnen, wenn der Zinkgehalt auf Spuren hinabgedrückt werden kann.

Der Vorsitzende: Ioh nehme an, daß Sie alle mit den Ausführungen des Hern Berichterstatters einverstanden sind.

Der Vorsitzende: Den nächsten Punkt der Tagesordnung bilden Fleischbrüh-Ersatzwürfel. Referent ist Herr Prof. Mai.

\title{
Suppen- und Brühwürfel.
}

\author{
V.on \\ C. Mai-München.
}

M. H.! Ich hatte die Absicht, Ihnen Vorschläge zur Beurteilung der Erzeugnisse der Würfelindustrie zu machen; bei der unmittelbar bevorstehenden Regelung dieser. Frage von Reichs wegen glaube ich indessen jetzt hiervon absehen zu sollen.

Trotzdem ist es aber vielleicht nicht überflüssig, einige kurze Bemerkungen über die Bezeichnung der verschiedenen. Würfel zu machen, da hierüber nicht allenthalben die erforderliche Klarbeit $\mathrm{zu}$ herrschen scheint.

Es ist zu unterscheiden zwischen „Suppenwürfel" und „Brühwürfel“. 